\title{
Le mythe herculéen dans trois séries américaines: Supernatural, Buffy the Vampire Slayer et The X-Files
}

\section{François-Ronan Dubois}

\section{OpenEdition}

\section{Journals}

Édition électronique

URL : http://journals.openedition.org/lcc/427

DOI : $10.4000 /$ lcc. 427

ISSN : 2430-4247

\section{Éditeur}

Université Aix-Marseille (AMU)

\section{Référence électronique}

François-Ronan Dubois, « Le mythe herculéen dans trois séries américaines : Supernatural, Buffy the Vampire Slayer et The X-Files », Les chantiers de la création [En ligne], 5 | 2012, mis en ligne le 26 novembre 2014, consulté le 08 avril 2020. URL : http://journals.openedition.org/lcc/427 ; DOI : https:// doi.org/10.4000/lcc.427 


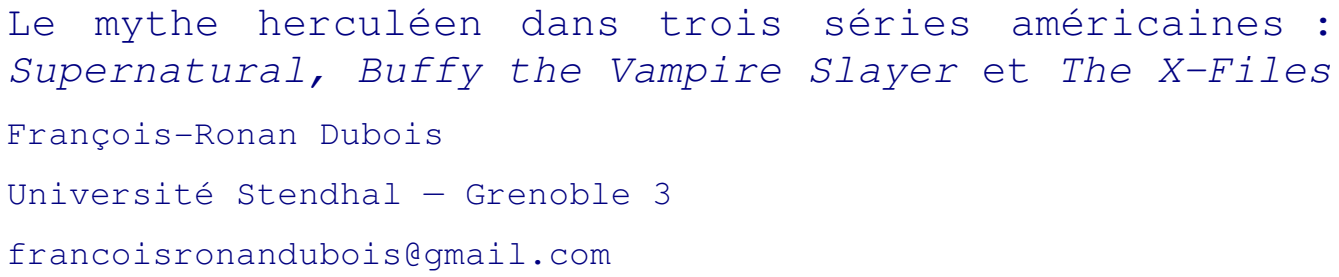

X-Files, Buffy, Supernatural, Mythocritique, Herakles

Historiquement, les études sur les séries télévisées ont été et sont encore façonnées par des influences académiques fort différentes. Ainsi les recherches consacrées à Buffy the Vampire Slayer peuvent-elles se répartir en cinquante domaines disciplinaires différents (Lavery 13). Si, d'un point de vue méthodologique, la validité de certaines catégories peut faire discussion ${ }^{1}$, il n'en demeure pas moins que l'étude des séries télévisées est un champ hétéroclite et particulièrement perméable aux méthodes importées d'autres domaines.

C'est donc à une distinction souple, qui vise uniquement à clarifier le présent propos, que je vais recourir ; il faudrait naturellement la discuter longuement et l'amender. Quoi qu'il en soit, je dirais ainsi que les études sur les séries télévisées ont pu avoir notamment trois types d'approches.

1) Très tôt, des études ont paru dont le propos était de décrire les réactions de tel ou tel public devant tel ou tel problème. Ainsi, en 1995, un article consacré à Hélène et les garçons affirmait-il que la série télévisée était «devenue un support central aux interactions interindividuelles quotidiennes » (Pasquier 13). Etudier les séries télévisées, c'est étudier des représentations sociales et les comportements qui les accompagnent.

2) D'une façon voisine, les séries télévisées sont un matériau de choix pour ce qui s'appelle parfois les études culturelles, consacrées aux questions de genre, de classes, de colonialisme, d'orientation sexuelle, etc. L'attitude est à la fois descriptive et militante. Ainsi dans un article consacré à Buffy: «But the right words and pictures can at the very

\footnotetext{
${ }^{1}$ Peut-on vraiment considérer, par exemple, que «Marxist » répond au même genre de distinction que «ethics»? Est-il pertinent de séparer «Philosophy» de «Feminist» (ou même «Foucaldian », qui équivaut, dans la classification de Lavery, à «Marxist») ? Les «Xander Studies », c'est-à-dire les études consacrées au personnage Xander, constituent-elles vraiment un ensemble indépendant? On voit que cette perméabilité n'est pas sans une certaine confusion méthodologique.
} 
least help to shape a revolution's canons. »(Karras, en ligne) $)^{2}$. Les personnages de série télévisée sont l'occasion de critiquer ou de soutenir, par une discussion universitaire argumentée, tel ou tel système de valeurs.

3) Plus traditionnellement, il a pu être question de discuter la manière dont les thèmes, les représentations culturelles, étaient construits. Par exemple, qu'est-ce qu'un vampire ? Ces discussions, plus traditionnelles en un sens, font appel à la variété des approches en recherches littéraires et cinématographiques.

C'est indubitablement dans cette troisième catégorie, celle de l'étude des thèmes, que se place le présent propos. En m'inspirant de la méthode évoquée par Gilbert Durand, à partir de Gaston Bachelard, dans les Structures anthropologiques de l'imaginaire, telle qu'elle a été depuis comprise par des chercheurs comme Philippe Walter, je propose d'examiner la survivance d'une structure mythique dans un corpus composé de trois séries télévisées et, plus particulièrement, des deux premières saisons ${ }^{3}$ de chacune d'entre elles. En d'autres termes, je fais le pari que l'on peut retrouver, entre les trois séries, une structure commune qui est aussi celle d'un mythe déjà ancien. Chaque série et chaque version $\mathrm{du}$ mythe constituent alors une manifestation particulière d'une structure fondamentale stable, manifestation qui peut être plus ou moins fidèle en cela qu'elle incorpore plus ou moins d'éléments de la version la plus ancienne. Ces éléments, qui sont à la fois des motifs narratifs et des noyaux de sens, nous les appellerons des mythèmes. Il s'agit alors de comparer les mythèmes que les textes partagent, le traitement qu'ils en font, ou bien, au contraire, d'identifier ceux qui manquent en tel ou tel endroit. Bien entendu, cette entreprise est essentiellement descriptive mais, pour en faciliter l'appréhension, je suggérerai des pistes d'interprétation.

Dans un premier temps, il s'agira de rappeler à grands traits le mythe d'Héraclès, tel qu'il se trouve dans la Bibliothèque historique de Diodore de Sicile ${ }^{4}$ et d'en isoler une série de mythèmes. Ce sont ces mythèmes que l'on cherchera par la suite à identifier dans chacune des trois séries successivement, pour observer les recouvrements et les manques.

\section{La Bibliothèque historique}

Notre attention au texte de Diodore de Sicile $^{5}$ sera nécessairement réduite. Ce qui nous intéresse, ce n'est bien sûr pas la lettre, ni même l'organisation narrative ou la visée idéologique, mais simplement la structure d'un mythe. De ce point de vue, extraire des

\footnotetext{
2 « Mais les bons mots et les bonnes images peuvent, tout du moins, aider à définir les cadres d'une révolution. »

${ }^{3}$ Plusieurs raisons président à cette restriction. S'agissant de Buffy et des X-Files, elle est simplement destinée à faciliter le résumé des intrigues en évitant les complications excessives qui interviennent inévitablement à mesure que l'histoire se développe, ceci afin d'assurer la bonne intelligence du propos par un lecteur qui, peut-être, n'aura pas tout vu. Le cas de Supernatural est plus complexe, dans la mesure où les deux premières saisons se distinguent, structurellement, des saisons suivantes : ce qui est valable pour cellesci, du moins en ce qui concerne le présent propos, ne l'est pas nécessairement pour celles-là.

${ }^{4} \mathrm{~J}$ 'emploie la Bibliothèque historique non en affirmant qu'il s'agirait de la version la plus pure ou la plus complète du mythe mais plutôt en songeant qu'elle est la plus synthétique, la plus suivie et, surtout, la plus aisée à se procurer. Pour aider encore le lecteur qui désirerait s'approprier le texte, j'utiliserai une version librement disponible en ligne, ce qui ne préjuge rien, naturellement, de la qualité des supports plus traditionnels.

${ }^{5}$ De Diodore de Sicile lui-même, il y a peu à dire, sinon qu'il vécut au premier siècle avant l'ère chrétienne et qu'il écrivit en grec.
} 
mythèmes de cette narration, c'est en quelque sorte réduire le texte en miettes; mais ce sont ces miettes et non le texte que nous manipulons. C'est pour cette même raison que je n'offrirai qu'un résumé très succinct, et même lacunaire, de l'existence d'Héraclès telle que Diodore la décrit.

Héraclès est le fils de Zeus et d'Alcmène, l'épouse d'Amphytrion. Pour abuser la fidélité de cette dernière, le roi des Dieux a pris l'apparence de son époux et allonge la durée de la nuit pour goûter plus longtemps à sa compagnie. A l'enfant qui naîtra de cette union, Zeus promet l'empire sur le royaume. Cette promesse suscite la jalousie d'Héra, qui prolonge la grossesse d'Alcmène et fait naître avant terme Eurysthée, qui devient l'héritier du pouvoir. Pour dédommager Héraclès, Zeus décide de lui proposer, en son âge adulte, douze travaux qui le porteront aux cieux. Alcmène, effrayée par la colère d'Héra, expose l'enfant. Héra, sans le savoir, recueille le nourrisson et lui donne sein : il mord trop fort et, de la poitrine de la déesse, du lait s'échappe qui forme la Voie Lactée. Courroucée, Héra envoie deux serpents tuer l'enfant, qui les étouffe sans difficulté. Héraclès grandit à Thèbes où son père Amphytrion est exilé : il surpasse, sans difficulté, tous ses camarades. C'est alors que survient sa première guerre, contre Erginus, roi des Myniens. Par la suite, Héraclès participe à de nombreuses guerres qui, parallèlement aux Travaux à proprement parler, constituent une part importante du récit de Diodore. Les Travaux sont quant à eux proposés à Héraclès par Eurysthée. Après avoir consulté l'Oracle de Delphes, le héros accepte la proposition du roi, assuré qu'il accomplit ainsi la volonté de Zeus. Héraclès accomplit avec succès ces Travaux ${ }^{6}$ et est engagé dans de multiples aventures, parfois amoureuses. C'est la passion qui lui coûte finalement la vie, quand son épouse Déjanire, jalouse des sentiments qu'il a pour Iolé, lui fait présent d'une tunique empoisonnée par le sang du centaure Nexus. Voué à une mort certaine, Héraclès érige un bûcher sur le mont Oeta. Après sa mort, il connaît l'apothéose.

Plusieurs aspects distinguent Héraclès non seulement du commun des mortels mais également des guerriers valeureux ou des brigands farouches qu'il affronte. Ce qui fait fondamentalement d'Héraclès un être à part, c'est d'abord sa naissance divine et l'intérêt que prennent, perpétuellement, les dieux à sa destinée :

Quoique Bacchus et Hercule fussent nés de femmes mortelles, ils furent cependant honorés du nom d'Olympiens, non seulement parce qu'ils étaient fils de Jupiter, mais aussi parce que, obéissant aux penchants de leur père, ils avaient adouci par leurs bienfaits la vie des hommes. (Diodore, 4,15 )

Cette origine divine, on le voit, a plusieurs conséquences : d'abord, Héraclès est un être distinct du commun des mortels, ensuite il se comporte d'une manière particulière (en accord avec les volontés de son père divin) et enfin il le fait dans une intention précise (pour adoucir la vie de l'humanité). L'habileté et la force surhumaines d'Héraclès ne sont donc pas purement destinées à ses accomplissements personnels mais servent la collectivité : le héros a un rôle civilisateur, qui peut prendre la forme d'une activité pacificatrice ou agricole. La cohérence de ces différentes activités est décrite lors du séjour du héros en Libye :

\footnotetext{
${ }^{6}$ Il s'agit, souvenons-nous en, d'étouffer le Lion de Némée à la peau impénétrable, de tuer l'Hydre de Lerne aux nombreuses têtes, de battre à la course la biche de Cérynie, animal d'Artémis, de capturer vivant le monstrueux sanglier d'Erymanthe, de nettoyer les écuries d'Augias (et il était temps), de tuer les innombrables oiseaux du lac Stymphale, de dompter le taureau de Crète, de capturer les juments anthropophages de Diomède, de voler la ceinture de la reine des Amazones Hippolyte, de s'emparer du troupeau des bœufs de Géryon, de cueillir les pommes d'or du jardin bien gardé des Hespérides et d'enchaîner Cerbère aux enfers.
} 
Hercule purifia ensuite la Libye d'un grand nombre d'animaux sauvages dont elle était remplie ; il fit cultiver beaucoup de contrées désertes, qui se couvrirent bientôt d'arbres fruitiers, de vignes, d'oliviers et d'autres plantations. En un mot, de la Libye, infestée de bêtes féroces, il fit une terre fertile et prospère ; exterminant les scélérats ou les despotes insolents, il rendit les villes florissantes. (Diodore, 4, 17)

Tuer des monstres n'est donc pas une exigence spécifique à la dynamique des Travaux imposés par Eurysthée : cette mission relève de l'activité quotidienne d'Héraclès, de son rôle civilisateur. Ces monstres peuvent être les créatures merveilleuses qu'il affronte lors des Travaux, les bêtes féroces de la Libye ou bien des brigands sans foi ni loi :

Il rendit la route, de rude et difficile qu'elle était, accessible à une armée avec tout son bagage. Les Barbares qui habitaient ces montagnes avaient coutume de piller et de massacrer les troupes qui les traversaient. Hercule soumit cette nation, et, après avoir puni les chefs des brigands, il assura pour toujours la sécurité de ces passages. (Diodore, 4, 19)

Il faut comprendre ici que l'activité du Tueur de Monstres a deux conséquences : elle pacifie le pays qu'ils infestaient et permet la circulation des voyageurs. Dans la mesure où les monstres, les brigands et les Géants ont la fâcheuse habitude de défier au combat tous ceux qui se présentent sur leur territoire, les contrées méditerranéennes vivent dans l'isolement; l'intervention du Tueur de Monstres permet au contraire de rétablir les relations commerciales. C'est que pour aller capturer les bœufs de Géryon, qui se trouvent sur la péninsule ibérique, Héraclès entreprend un long voyage par l'Afrique du Nord, vers l'Espagne, avant de remonter la péninsule, de passer en Gaule et de revenir, par ce chemin, en Grèce ; Héraclès est également un héros voyageur :

Il n'est pas raisonnable que les hommes envient à Hercule les louanges dues aux bienfaits de la civilisation qu'il a répandus sur la terre par tant de travaux. (Diodore de Sicile, 4, 8)

Ce n'est donc pas la seule Grèce qu'Héraclès civilise, mais la terre entière, c'est-à-dire l'espace imaginaire grec qui embrasse l'ensemble de la Méditerranée. Cette vie nomade lui permet de rapporter des savoirs étrangers :

Comme Hercule apporta le premier en Grèce la science de la sphère, il en retira une grande gloire ; c'est ce qui fit dire aux hommes, allégoriquement, qu'il avait reçu d'Atlas le fardeau du monde. (Diodore de Sicile, 4, 27)

Le rôle d'Héraclès dans la circulation des connaissances est donc double : certes, il exporte hors de Grèce les connaissances agricoles, détourne des fleuves, favorise les cultures, mais il importe également dans son pays des savoirs mathématiques.

Rassemblons nos informations. Héraclès est un héros civilisateur, qui construit un monde imaginaire commun en voyageant, qui le domestique en le purgeant des monstres, grâce à son savoir, à son habileté guerrière et à la protection des Dieux. Je propose de résumer en six mythèmes fondamentaux les aspects du mythe civilisateur herculéen :

1) Naissance divine et destin,

2) Habileté et force surhumaines,

3) Voyage,

4) Rôle civilisateur,

5) Tueur de monstres,

6) Savoir.

Ce sont ces mythèmes que nous allons à présent essayer de retrouver dans les séries télévisées. 


\section{Trois séries américaines : présentation et analyse}

Avant d'examiner successivement les trois séries télévisées, il faut leur remarquer quelques parentés. Certaines de ces parentés ont été brièvement évoquées par Stacey Abbott : Buffy et X-Files seraient deux séries fondatrices d'un nouveau genre télévisuel entre horreur et fantastique, qui a tracé la route pour Supernatural. La bibliographie académique sur Buffy, la plus étendue des trois de notre corpus, mentionne encore en diverses occasions $X$-Files qui, à la même époque que Buffy, partage un bestiaire parfois voisin. ${ }^{7}$ Pour résumer à grands traits, disons que nous avons affaire à trois séries américaines (très) populaires, mettant en scène des héros aux prises avec des monstres fantastiques.

Il serait d'usage d'envisager ces séries dans un ordre chronologique, dans la mesure où Supernatural semble être la conséquence culturelle de Buffy et de X-Files (entre autres), mais puisque notre propos est un problème de structure et non d'histoire, il semble plus profitable de traiter les manifestations du mythe selon leur plus ou moins grande conformité au texte le plus ancien. J'examinerai donc dans un premier temps Supernatural, puis Buffy et enfin The X-Files.

\subsection{Supernatural}

Supernatural est une série télévisée américaine qui compte actuellement 7 saisons et 138 épisodes d'une quarantaine de minutes chacun. Créée par Eric Kripke, elle débute en 2005 sur le WB avant de migrer sur le CB, né de la réunion du WB et de l'UPN.

L'histoire est essentiellement consacrée au voyage en voiture (road trip) accompli dans l'ensemble des Etats-Unis d'Amérique, Dean et Sam Winchester ${ }^{8}$. Quand Dean avait une dizaine d'années et Sam exactement six mois, leur mère Mary ${ }^{9}$ a été tuée par une créature plus tard appelée le Démon aux Yeux Jaunes (Yellow-Eyed Demon). Le père des deux garçons, John ${ }^{10}$, les a donc élevés en marge de la société en leur apprenant à chasser les monstres : vampires, démons, loups-garous, fantômes, dieux anciens. Mais voilà que, alors qu'ils ont une vingtaine d'années, leur père disparait. Sam, qui prévoyait de devenir avocat, abandonne ses études, comme l'y incite le meurtre de sa fiancée ${ }^{11}$, victime à son tour du Démon aux Yeux Jaunes, et accompagne son frère dans ses recherches. Fort

\footnotetext{
${ }^{7}$ Il arrive qu'une série fasse référence à une autre. Ainsi Buffy dit-il à Giles : « I cannot believe that you of all people are trying to Scully me » (Je n'arrive pas à croire que vous, spécialement vous, essayiez de me scullyfier), faisant référence ainsi à l'esprit positif de l'hérö̈ne de X-Files (« The Pack », Saison 1, Episode 6). De la même façon, dans l'épisode «The Usual Suspects » (Saison 2, Episode 7), les deux héros, Sam et Dean, se disputent en ces termes :

«DEAN. What do you think, Scully? You wanna check it out? (Qu'est-ce tu en penses, Scully ? Tu veux aller vérifier?)

SAM. I'm not Scully, you're Scully. (Je ne suis pas Sculy : c'est toi.)

DEAN. No, I'm Mulder. You're a red-headed woman. » (Non. Je suis Mulder. C'est toi, la femme rousse.)

${ }^{8}$ Respectivement Jensen Ackles et Jared Padalecki.

${ }^{9}$ Samantha Smith généralement.

${ }^{10}$ Jeffrey Dean Morgan.

${ }^{11}$ Adrianne Palicki.
} 
habiles en leur domaine, les deux frères purgent les Etats-Unis d'un grand nombre de créatures et éventent les plans du Démon aux Yeux Jaunes : ce dernier contamine des enfants de son sang, afin d'acquérir sur eux un ascendant psychologique. Son but ultime est d'ouvrir une Bouche des Enfers, protégée par une barrière mystique forgée par Samuel Colt et que les démons ne peuvent franchir. Les frères Winchester, dans leur lutte, remportent une victoire à la Pyrrhus : s'ils parviennent à occire leur ennemi grâce à un Colt magique ils ne parviennent pas à fermer la Bouche des Enfers à temps, qui a permis à de nombreux démons de s'enfuir. Ils ont donc bien des travaux devant eux à l'issue de la deuxième saison.

L'un des traits distinctifs de la série est indubitablement son ancrage géographique : délaissant les grandes villes modernes, les frères Winchester chassent généralement dans les petites villes du Middle West, parfois durement frappées par la crise industrielle (Tovar). Les Winchester sont comparés à des cow-boys et leur road trip est profondément ancré dans l'esthétique du western : ils arrivent dans une petite ville, tuent le fauteur de troubles local, embrassent la jolie jeune fille et reprennent la route. 


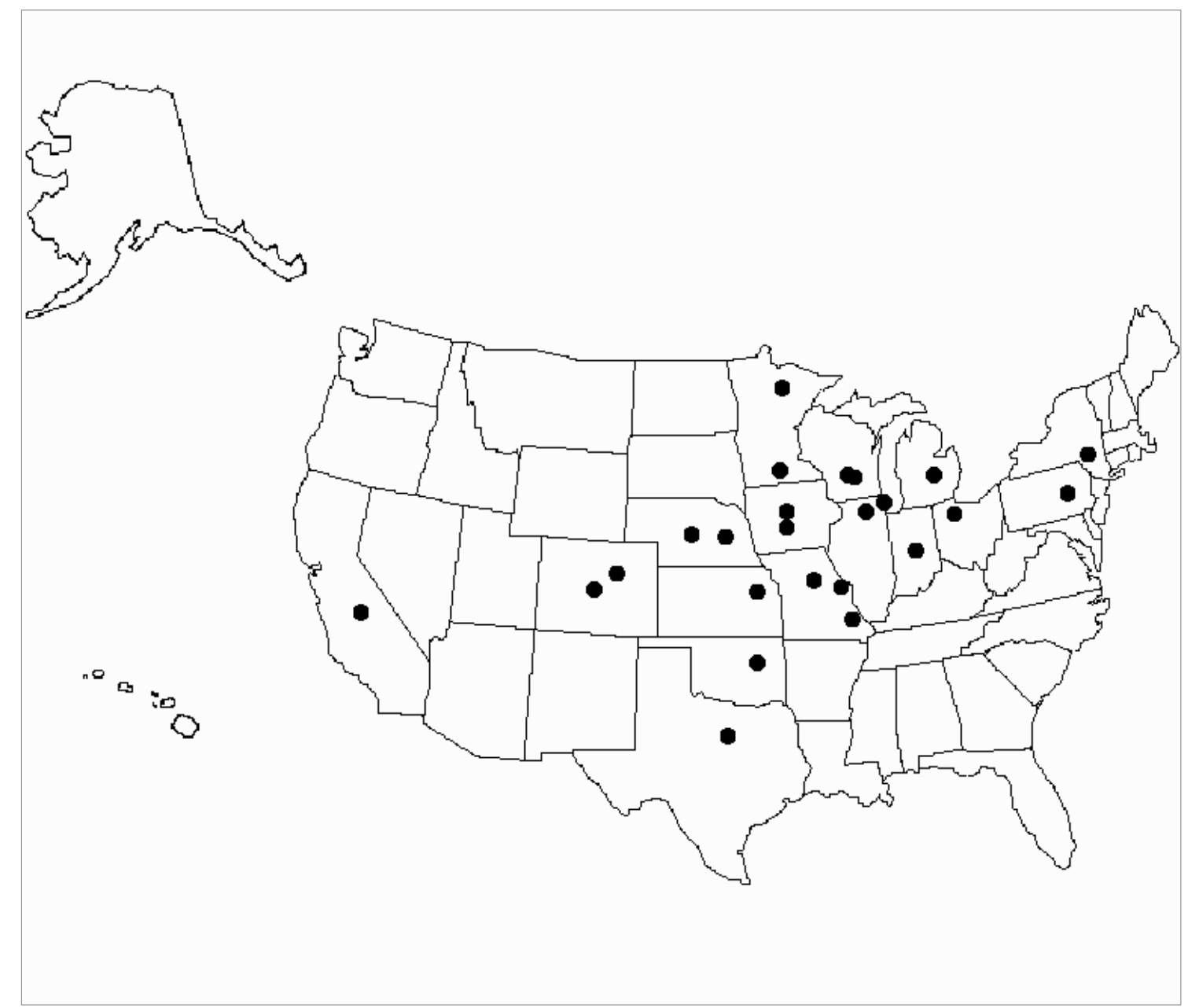

Carte des emplacements de la première saison. Les lieux fictionnels sont indiqués par une marque au centre de l'Etat (Etat réel, ville imaginaire). 


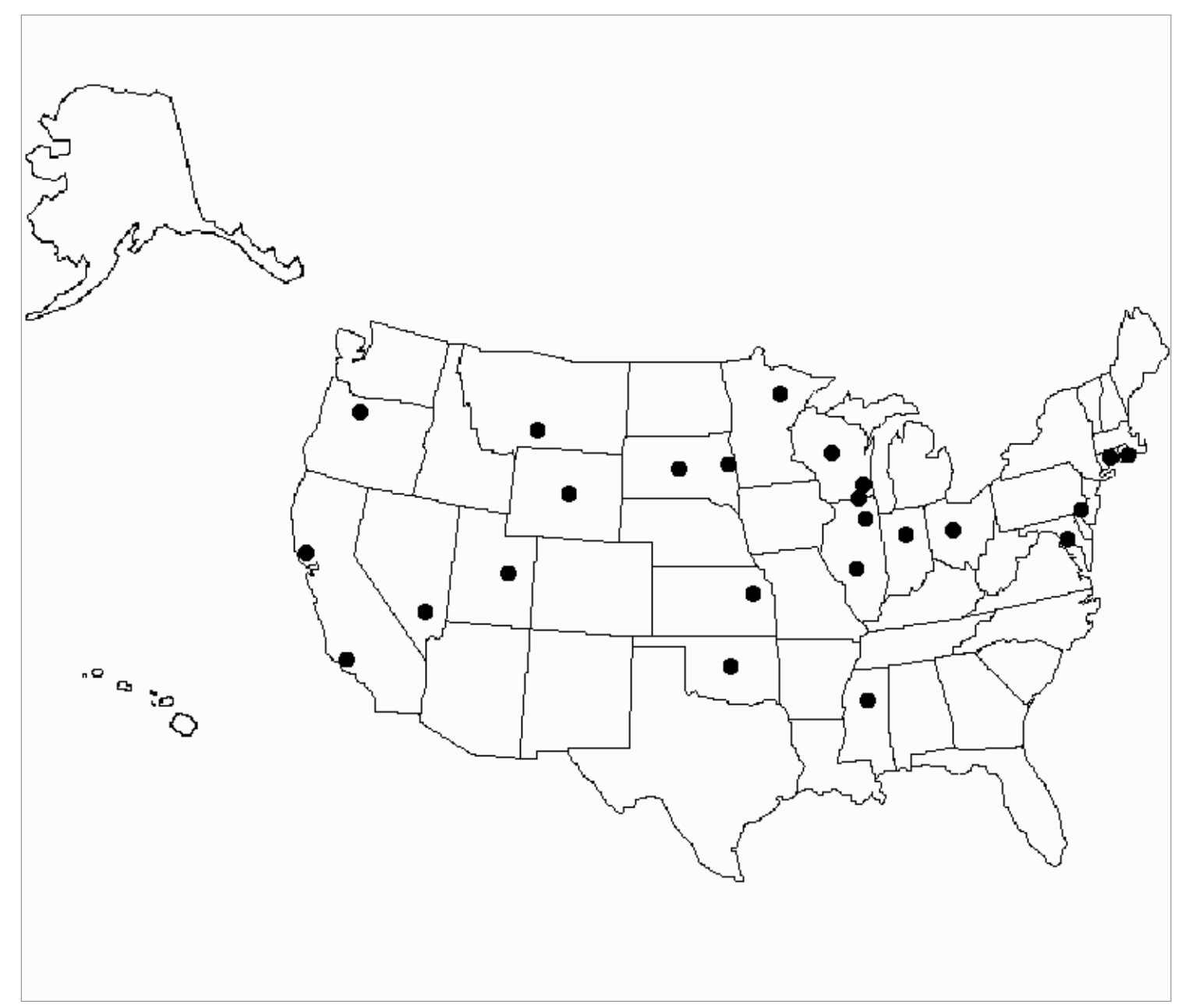

Carte des emplacements de la deuxième saison. Les lieux fictionnels sont indiqués par une marque au centre de l'Etat (Etat réel, ville imaginaire).

Si les frères Winchester sont en mesure de restaurer ainsi la tranquillité des petites villes et, en de nombreuses occasions, de permettre aux habitants de circuler plus librement, c'est à la fois grâce aux exceptionnelles compétences de chasseurs et de combattants et grâce aux notes que John Winchester a laissées à ses fils.

$\mathrm{Si}$ nous tentons de formuler cette histoire fondamentale en mythèmes pour en dégager la structure et si, plus particulièrement, nous tentons de la reformuler dans les mêmes termes que ceux que nous avons utilisés pour décrire le mythe herculéen, nous obtenons la liste suivante:

1) Naissance et destin démoniaque pour Sam

2) Habileté et force surhumaines pour les deux frères

3) Voyage

4) Rôle civilisateur

5) Tueurs de monstres

6) Savoir occulte

Nous constatons que quatre des six mythèmes correspondent convenablement à la structure du mythe herculéen : les compétences physiques, le voyage, le rôle civilisateur et les tueurs de monstres. En revanche le premier et le dernier mythèmes sont, en quelque sorte, des anti-mythèmes : Sam n'a pas de destinateur de divin (comme Héraclès Zeus) mais un anti-destinateur démoniaque et le savoir des deux héros n'est ni partagé ni positif mais occulte et hermétique. 
Si nous avons correctement ordonné le corpus, nous devons nous attendre à de nouvelles perturbations avec les séries suivantes.

\subsection{Buffy the Vampire Slayer}

Avec deux revues scientifiques presque exclusivement consacrées à son étude, Slayage : The Journal of the Whedon Studies Association et Watchers Juniors : The Undergraduate Journal of Whedon Studies, Buffy jouit, depuis plus de dix ans, d'une considérable attention critique, qui en fait sans aucun doute la série la plus représentée au sein de l'université. Créée en 1997 par Joss Whedon ${ }^{12}$, diffusée d'abord en Nouvelle-Zélande pendant quelques semaines puis dans son pays d'origine sur les mêmes chaînes que Supernatural, Buffy compte, à la fin de sa diffusion en 2003, 143 épisodes d'une quarantaine de minutes pour 7 saisons.

La série évoque l'histoire de Buffy Summers ${ }^{13}$, lycéenne au dossier scolaire chargé, qui s'installe dans la petite ville en apparence paisible de Sunnydale. Mais Sunnydale est malheureusement située au-dessus d'une Bouche des Enfers et de nombreux monstres, au premier rang desquels des vampires, déferlent la nuit dans les rues pour attaquer les habitants. Par chance, Buffy est la Tueuse, l'élue des Forces du Bien dans leur lutte contre les Forces du Mal. Aidée par ses amis Willow et Xander, ainsi que par le Gardien et formateur Giles ${ }^{14}$, accessoirement bibliothécaire du lycée, Buffy va d'abord déjouer les plans du Maître ${ }^{15}$, vampire ancestral, et rencontrer l'amour en la personne d'Angel ${ }^{16}$, vampire à l'âme torturée. Mais Angel perd son âme et, dans la deuxième saison, mène la vie rude à Buffy et ses acolytes. C'est qu'Angel prévoit, avec l'aide de Drusilla, vampire à l'état mental plus que douteux, de libérer un démon qui avalera le monde et provoquera l'Apocalypse. Alors que Willow s'efforce de restaurer l'âme d'Angel pour le ramener à de meilleurs sentiments, Buffy se résout à une alliance avec Spike, vampire cruel mais désireux de contrecarrer les plans d'Angel pour regagner le cœur de Drusilla ${ }^{17}$. Au moment où Willow parvient à rendre son âme à Angel, Buffy est contrainte de le tuer pour fermer le vortex interdimensionnel qui menace de détruire le monde. La victoire est amère.

Remarquable pour sa présentation humoristique de certains éléments fantastiques ${ }^{18}$ et pour son mélange alors novateur du teen drama et du horror show, la série se distingue avant tout par la variété de son bestiaire. Le rôle de Buffy, dans cet univers, est très clairement identifié et il est longuement décrit, en de multiples occasions, par des prophéties ou par les considérations amères des démons qu'elle s'apprête à défaire. C'est que l'élection divine de Buffy n'est pas anodine : la jeune femme possède une force, une agilité, une vitesse et une maîtrise des armes très supérieures à celles des humains et de bien des créatures de la nuit. Quelques visions viennent même ajouter à la liste de ses capacités. Ces facultés bien utiles ne l'en dispensent pas moins de mener de longues

${ }^{12}$ Par ailleurs célèbre pour sa série space-western intitulée Firefly ainsi que pour le spin-off de Buffy, Angel.

${ }^{13}$ Sarah Michelle Gellar.

${ }^{14}$ Nicholas Brendon, Alyson Hannigan et Anhtony Stewart Head.

${ }^{15}$ Mark Metcalf.

${ }^{16}$ David Boreanaz.

${ }^{17}$ James Marsters et Juliet Landau.

${ }^{18}$ Dont la phrase suivante de Giles résume assez bien l'esprit : «Uh, let me make sure I have this right. This witch is casting horrible and disfiguring spells so that she can become a cheerleader ? (Attendez que je sois sûr de bien comprendre. Une sorcière lance d'affreux sorts défigurants pour pouvoir devenir cheerleader ?) («Witch », Saison 1, Episode 3) 
recherches en bibliothèque pour s'informer de son ennemi ou, bien souvent, de déléguer cette fonction à ses amis, avec lesquels il faut compter (Wandless, Bertho). Au-delà de l'ennemi ponctuel qui sert de support à la plupart des épisodes particuliers, Buffy et ses amis sont engagés dans une lutte contre le grand ennemi de la saison, dont le dessein est souvent l'anéantissement du monde ou tout du moins la souffrance des pauvres gens : Buffy cherche ainsi à préserver la quiétude de Sunnydale en particulier et de notre dimension en général.

Nous pouvons à présent tenter de nous livrer à l'identification des mythèmes :

1) Naissance et destination divines

2) Habileté et force surhumaines

3) Sunnydale

4) Rôle civilisateur

5) Tueuse de monstre

6) Savoir occulte

A nouveau, quatre mythèmes satisfaisants ${ }^{19}$ et deux manquants. Je ne reviens pas sur le savoir occulte, qui accueille les mêmes remarques que celles formulées sur Supernatural. Du point de vue de la structure, il faut cependant remarquer que «Sunnydale » est un mythème qui incarne particulièrement le « Voyage » de Sam et Dean. Pour Sam et Dean, «Voyage » est un ensemble de «Sunnydale» : c'est le même genre de paysages, le même genre de villes.

Retenons ces identités et ces différences pour mieux aborder notre troisième et dernière série.

\subsection{The X-Files}

La vie de X-Files recouvre à peu près celle de Buffy. Créée en 1993 par Chris Carter, la série, diffusée sur la Fox jusqu'en 2002, compte 202 épisodes d'une quarantaine de minutes pour 9 saisons, ainsi que plusieurs films.

La série expose les péripéties de Fox Mulder et Dana Scully ${ }^{20}$, deux agents du FBI. Fox Mulder, brillant profiler formé en Grande-Bretagne, est convaincu que sa sœur Samantha a été enlevée par des extraterrestres lorsqu'ils étaient enfants et qu'une conspiration gouvernementale internationale dissimule l'existence des aliens au grand public ; il est par ailleurs disposé à prêter foi aux phénomènes paranormaux de toute sorte. Tout au contraire, Dana Scully, docteur en médecine, experte de médecine légale, femme de sciences, aborde ces étranges manifestations d'un œil positif. C'est d'ailleurs originellement sa mission : détruire par la preuve la crédibilité des affaires non-classées sur lesquelles travaille l'agent Mulder. Mais bientôt, une complicité se noue entre les deux collègues et Scully découvre, à son tour, des preuves de la sinistre conspiration. Elle est elle-même enlevée. Par qui ? Des extraterrestres? Le gouvernement secret? Des scientifiques japonais menant de sinistres expériences sur les citoyens américains ?

S'il est difficile de le savoir, c'est que X-Files se distingue par l'extrême complexité, du moins dans les premières saisons, de son intrigue. Si le spectateur peut être à peu près certain des groupes en présence ${ }^{21}$, il n'est jamais facile de décider qui fait quoi

\footnotetext{
${ }^{19}$ Il est à noter que Buffy a déjà été comparée à des héros mythiques en raison de ces caractéristiques très spécifiques : ainsi David Fritts a-t-il pu la comparer à Beowulf.

${ }^{20}$ David Duchovny et Gillian Anderson.

${ }^{21}$ Pour faire simple : les extraterrestres qui prévoient de coloniser la terre, le gouvernement secret qui aide les extraterrestres pour ménager un compromis, des hybrides extraterrestres (produits par le gouvernement secret?) qui s'opposent tant aux extraterrestres qu'au gouvernement secret (mais pas
} 
dans quel but et de nombreux épisodes font se succéder les hypothèses pour n'en valider aucune. C'est dans ce contexte qu'interviennent des épisodes plus purement fantastiques où les agents Mulder et Scully côtoient les vampires, les sorcières et les monstres de toute sorte. Le mode opératoire est alors souvent le même : l'agent Mulder a repéré, dans un journal local, une affaire étrange. Les deux agents prennent l'avion, louent une voiture, perdent parfois leur chemin, avant de se retrouver dans une petite ville fort typique de l'Amérique profonde où un shérif désemparé leur expose son désarroi. Armés l'une de la science, l'autre d'un savoir encyclopédie sur le paranormal, les deux agents traquent le monstre/mutant/produit d'une expérience scientifique ratée et, en de rares occasions, réussissent à le détruire. Qu'ils y parviennent ou non, la petite ville acquiert à la fin de l'épisode un sentiment (souvent trompeur, donc) de sécurité et les deux agents, plus perplexes qu'éclairés, quittent la chambre qu'ils avaient louée dans un motel miteux pour retourner à Washington, où leur hiérarchie kafkaïenne consultera leurs rapports d'un œil pour le moins perplexe.

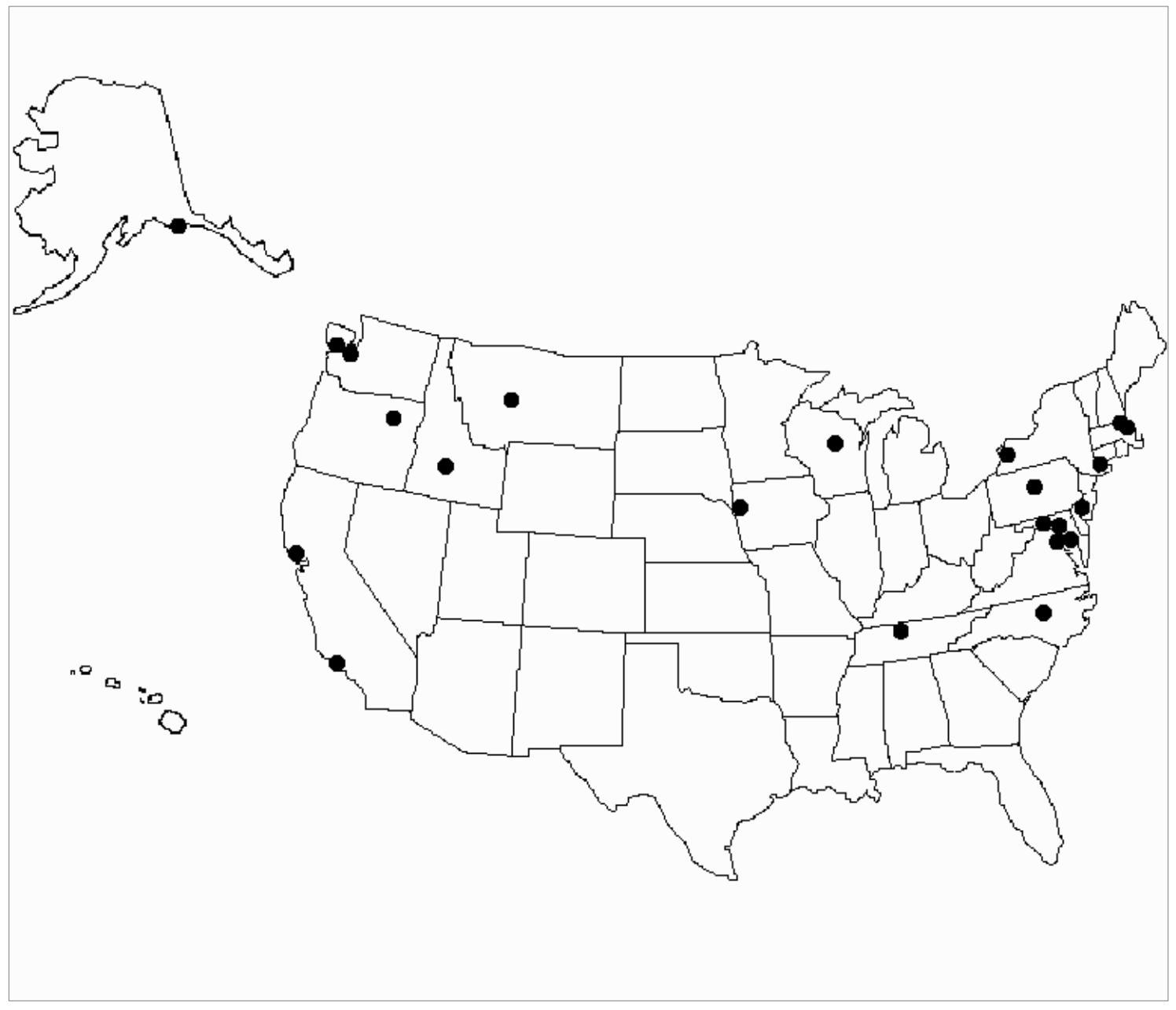

Carte des emplacements de la première saison

toujours), l'Homme à la Cigarette (William B. Davis) qui, au sein du gouvernement secret, poursuit ses propres buts (parfois), les informateurs de Mulder aux motivations souvent douteuses, le complexe militaroindustriel qui exploite les OVNIs pour dissimuler des essais militaires secrets et, pourquoi pas, un complot destiné à alimenter la machine de guerre américaine. Les militaires se font passer pour des extraterrestres, les extraterrestres pour des militaires, le gouvernement secret pour les deux et inversement. Tout cela sans compter, bien entendu, les divers scientifiques financés par le gouvernement secret (ou les hybrides ? ou les militaires ? ou le complexe militaro-industriel ?), qui parfois décident de trahir (on ne sait pas très bien qui). 


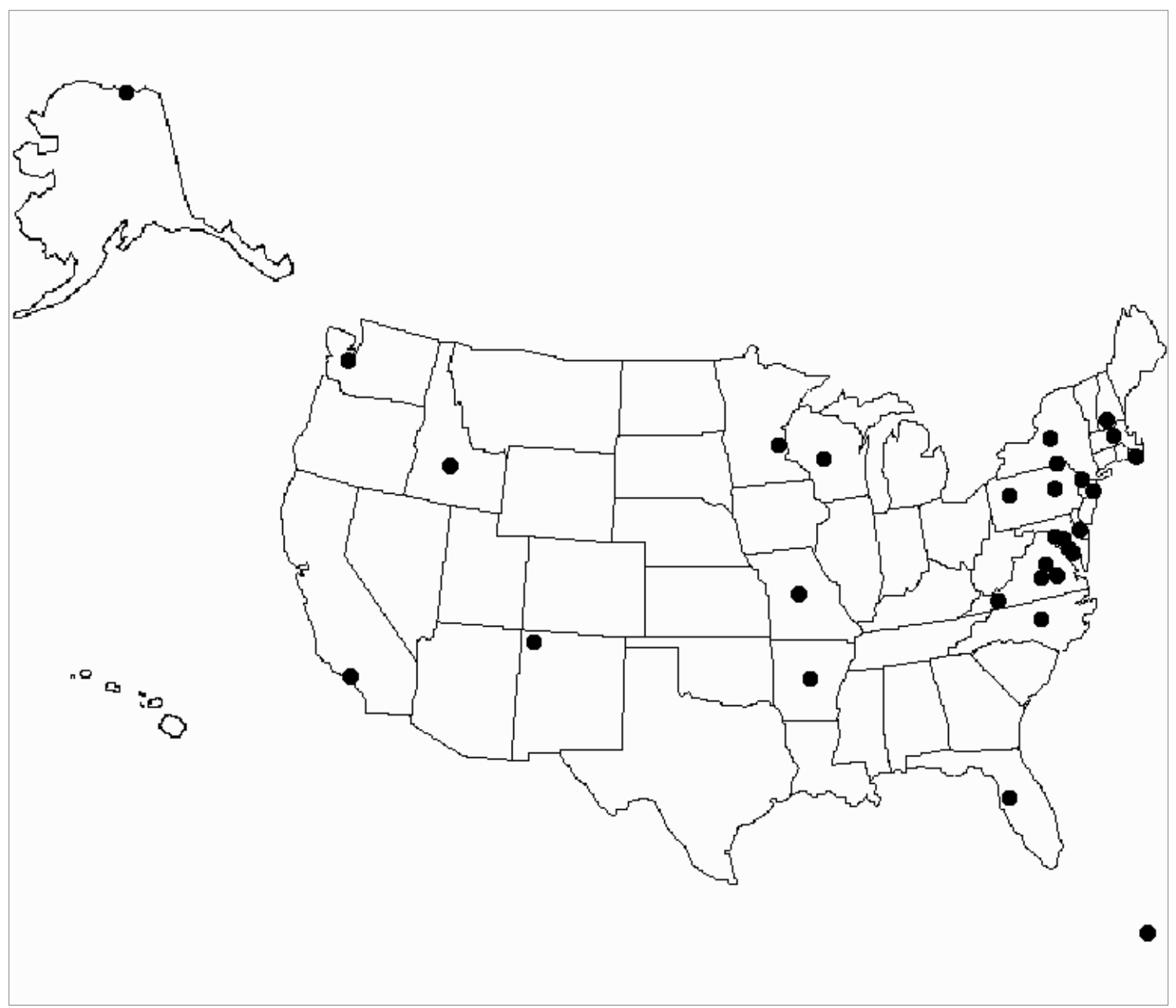

Carte des emplacements de la seconde saison 
L'identification des mythèmes est beaucoup plus complexe dans X-Files que dans les séries précédentes :

1) Motivé par l'enlèvement de sa sœur (Mulder) / Assignée aux affaires non-classées par la hiérarchie (Scully)

2) Habileté et force humaines

3) Voyage

4) Rôle civilisateur (rarement accompli)

5) Tueurs de monstres (parfois)

6) Savoir occulte (Mulder) / Savoir positif (Scully)

En dehors du voyage, chaque mythème, s'il est bien présent d'une certaine manière (sauf pour le deuxième), fait l'objet d'une négociation. Sans parler des mythèmes à demiréalisés, présents en intention plus qu'en effet (le quatrième et le cinquième), certains mythèmes renversent la structure du mythe. Ainsi le savoir positif de Scully, assimilable en tout point au savoir herculéen de la sphère ou de l'hydrologie, est-il souvent mis en défaut dans X-Files. Le savoir occulte de Mulder, certes plus fiable, n'a cependant pas l'efficacité de celui de John Winchester ou de Rupert Giles. Plus marquante encore est la question de la destination divine : c'est le gouvernement secret qui, on l'apprendra plus tard, a enlevé la sœur de Mulder et c'est encore le gouvernement secret qui assigne Scully aux affaires non-classées. En d'autres termes, le gouvernement secret remplit dans la structure herculéenne de $X$-Files le rôle du destinateur divin. L'ironie serait déjà assez cruelle si, d'un autre côté, ce n'était pas ce même gouvernement secret qui entravait la progression des héros et jouait donc le rôle d'anti-destinateur. Or, on le sait, le plan destinateur/antidestinateur est celui des valeurs : la confusion des rôles, même si elle n'est qu'apparente ${ }^{22}$, entraîne une confusion des valeurs. The X-Files présente donc une structure mythique à la limite de la rupture.

L'analyse descriptive grâce à la mythocritique structurale a permis de découvrir plusieurs éléments. D'abord, et c'est déjà quelque chose, que la mythrocritique structurale, telle que je l'ai présentée tout du moins, a une approche productive pour l'analyse des séries télévisées. Ensuite, qu'il persiste, dans la culture populaire américaine, une structure mythique fondamentale que nous pouvons comprendre grâce à un mythe qui nous est familier, celui d'Héraclès. Enfin, que cette structure mythique est l'objet de certains réaménagements récurrents (la petite ville, le savoir occulte, les anti-destinateurs en place de destinateurs) ou atypiques (la négation du savoir positif, l'indétermination des valeurs).

A partir de ces éléments, il faudrait bien sûr construire désormais une interprétation, ce qui relève déjà un peu d'une autre technique d'analyse. Je me contenterai donc de l'esquisser ici à très grands traits. Le motif de la petite ville me paraît être la clef pour bien comprendre ces réaménagements. Souvenons-nous que le rôle du mythe herculéen est explicitement, chez Diodore de Sicile, de construire une civilisation unifiée. Si ce mythe persiste dans la société américaine contemporaine, on peut supposer que c'est en raison d'une utilité profonde et il faut alors comprendre que l'espace américain n'est pas unifié. Si l'on songe désormais aux oppositions incessantes des agents Scully et Mulder, du FBI, c'est-à-dire de l'Etat fédéral, avec les forces de l'ordre locales, si l'on songe à la perpétuelle hostilité des petites villes de Sam et Dean Winchester, l'on découvre une

\footnotetext{
${ }^{22}$ Car c'est bien sûr la vérité qui, in fine, motive Scully et Mulder.
} 
opposition fondamentale entre l'Etat fédéral et les villes ${ }^{23}$, opposition politique et sociale profonde, dont le caractère conflictuel est incarné par les monstres, qui interdisent les échanges et la quiétude. Or, le savoir occulte, qui permet la résolution, est avant tout un savoir absent du corps social : il n'est ni agriculture, ni mathématiques. Faut-il y voir un problème dans la circulation des connaissances ? Encore une fois, ici se trouve le chantier de l'interprétation.

\section{Ouvrages cités}

Buffy the Vampire Slayer. 1993-2003. Télévision. Joss Whedon.

Supernatural. 2005-2012. Télévision. Eric Kripke.

The X-Files. 1993-2002. Télévision. Chris Carter.

Abbott, Stacey. «Then : The Road So Far ». TV Goes to Hell : An Unofficial Road Map of Supernatural. Toronto : ECW Press, 2010.

Badman, Derik A. «Academic Buffy Bibliography ». Slayage. №7 (2002). 20 février 2012 : < http://slayageonline.com/PDF/badman.pdf >

Bertho, Vanessa. «La bibliothèque dans Buffy contre les vampires: grimoires et merveilles». Conserveries mémorielles. N5 (2008). 20 février 2012: < http://cm.revues.org/84 >

Bradney, Anthony. «The Politics and Ethics of Researching the Buffyverse ». Slayage. N¹9 (2006). 20 février 2012 : < http://slayageonline.com/essays/slayage19/Bradney.htm >

Cantor, Paul A. «This Is Not Your Father's FBI : The X-Files and the Delegitimation of the Nation-State ». The Independent Review. Vol. 5, $\mathrm{n}^{\circ} 1,2001: 113-23$.

Durand, Gilbert. Les Structures anthropologiques de l'imaginaire. Paris : Dunod, 1960.

Fritts, David. «Warrior Heroes : Buffy the Vampire Slayer and Beowulf». Slayage. $\mathrm{N}^{\circ} 17$ (2005) : < http://slayageonline.com/PDF/fritts.pdf >

Karras, Irene. «The Third Wave's Final Girl : Buffy the Vampire Slayer ». Thirdspace. $\mathrm{N}^{\circ} 1.1$ (2002) : < http://www.thirdspace.ca/journal/article/view/karras/50 >

Lavery, David. "'I wrote my thesis on you !' : Buffy Studies as an Academic Cult». Slayage. $\mathrm{N}^{\circ} 14(2004):<\mathrm{http}: / /$ slayageonline.com/essays/slayage13_14/Lavery.htm >

Pasquier, Dominique. "'Chère Hélène' : les usages sociaux des séries collèges ». Réseaux. Nº70, 1994 : 9-39.

\footnotetext{
23 Sur la traitement de l'Etat fédéral dans The X-Files, on peut lire avec profit l'article de Paul Cantor cité en bibliographie, qui me paraît applicable à bien des séries policières, fantastiques ou non, de ces dix dernières années ; il y a là une importante question sur laquelle il faudra revenir.
} 
Tovar, Elisabeth. 7 novembre 2011. «Supernatural 'small-town America' : errance hantée dans les vestiges de l'Amérique industrielle ». Métropolitiques. 23 février $2012:<$ http://metropolitiques.eu/Supernatural-small-town-America.html >

Wandless, William. «Undead Letters : Searches and Researches in Buffy the Vampire Slayer ». Slayage. $\mathrm{N}^{\circ} 1$ (2001) : < http://slayageonline.com/PDF/wandless.pdf >

Wilcox, Rhonda V. "In 'The Demon Section of the Card Catalog' : Buffy Studies and Television Studies». Slayage. N N 21 (2006). 20 février 2012: < http://slayageonline.com/essays/slayage21/Wilcox.htm > 